\title{
Callyspongiolide is a Potent Inhibitor of the Vacuolar ATPase
}

Sarah Fogarty,$\ddagger$ Yeyun Ouyang, ${ }^{\dagger}$ Liubo Li,$\dagger$ Yu-Chan Chen,,${ }^{\dagger}$ Hallie Rane,${ }^{\perp}$ Francesco Manoni, ${ }^{\dagger}$ Karlett J. Parra,,${ }^{\perp}{ }^{*}$ Jared Rutter, $,{ }^{\ddagger},{ }^{*}$ and Patrick G. Harran ${ }^{\dagger, *}$

${ }^{\dagger}$ Department of Chemistry and Biochemistry, University of California-Los Angeles, 607 Charles E. Young Drive East, Los Angeles, California 90095-1569, United States

*Department of Biochemistry, University of Utah School of Medicine, Salt Lake City, Utah 84132, United States ${ }^{\S}$ Howard Hughes Medical Institute, University of Utah School of Medicine, Salt Lake City, Utah 84132, United States

${ }^{\perp}$ Department of Biochemistry and Molecular Biology, School of Medicine, University of New Mexico Health Sciences Center, Albuquerque, New Mexico, 87131, United States

\section{Supporting Information of Synthetic Compounds}

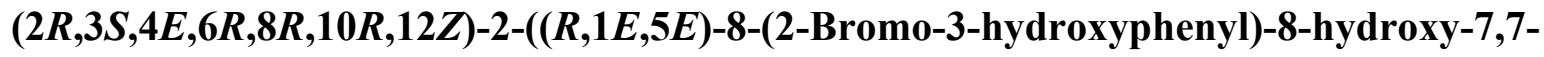
dimethylocta-1,5-dien-3-yn-1-yl)-3,6,10-trimethyl-14-oxooxacyclotetradeca-4,12-dien-8-yl carbamate, (-)-callyspongiolide (1)

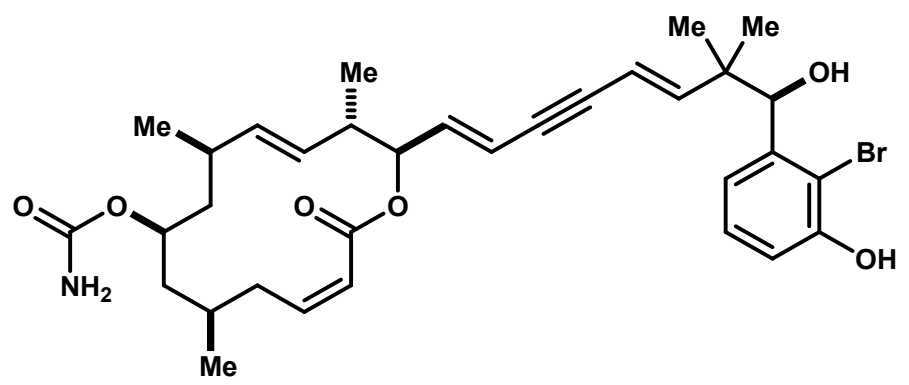

White Solid, $[\alpha]_{\mathrm{D}}=-13.1(c=0.1, \mathrm{MeOH}) .{ }^{1} \mathrm{H} \mathrm{NMR}\left(500 \mathrm{MHz}, \mathrm{CDCl}_{3}\right): \delta=7.24$ (app. t, $\left.1 \mathrm{H}\right)$, $7.03(\mathrm{dd}, 1 \mathrm{H}, J=1.5,7.8 \mathrm{~Hz}), 6.98(\mathrm{dd}, 1 \mathrm{H}, J=1.5,8.0 \mathrm{~Hz}), 6.35(\mathrm{~d}, 1 \mathrm{H}, J=16.3 \mathrm{~Hz}), 6.12(\mathrm{td}$, $1 \mathrm{H}, J=3.4,12.2 \mathrm{~Hz}), 6.02(\mathrm{dd}, 1 \mathrm{H}, J=8.1,15.8 \mathrm{~Hz}), 5.91-5.85$ (m, $2 \mathrm{H}), 5.67$ (s, $1 \mathrm{H}), 5.55$ (dd, $1 \mathrm{H}, J=2.1,16.3 \mathrm{~Hz}), 5.32(\mathrm{dd}, 1 \mathrm{H}, J=9.5,15.0 \mathrm{~Hz}), 5.27(\mathrm{dd}, 1 \mathrm{H}, J=8.5,10.5 \mathrm{~Hz}), 5.05(\mathrm{dd}$, $1 \mathrm{H}, J=9.3,15.0 \mathrm{~Hz}), 5.00$ (d, $1 \mathrm{H}, J=3.2 \mathrm{~Hz}$ ), 4.60 (app. t, $1 \mathrm{H}), 4.45$ (br. s, $2 \mathrm{H}), 3.73-3.63$ (m, $1 \mathrm{H}), 2.29-2.18(\mathrm{~m}, 1 \mathrm{H}), 2.15-2.05(\mathrm{~m}, 1 \mathrm{H}), 2.01-1.94(\mathrm{~m}, 1 \mathrm{H}), 1.92$ (d, $1 \mathrm{H}, J=3.6 \mathrm{~Hz}), 1.91$ $1.83(\mathrm{~m}, 1 \mathrm{H}), 1.54-1.45$ (m, $2 \mathrm{H}), 1.14$ (s, 3 H), 1.13-1.04 (m, 2 H), 1.05 (s, 3 H), 1.01 (d, 3 H, $J$ $=7.0 \mathrm{~Hz}), 0.96(\mathrm{~d}, 3 \mathrm{H}, J=6.8 \mathrm{~Hz}), 0.92(\mathrm{~d}, 3 \mathrm{H}, J=6.8 \mathrm{~Hz}) \cdot{ }^{13} \mathrm{C} \mathrm{NMR}\left(125 \mathrm{MHz}, \mathrm{CDCl}_{3}\right): \delta=$ $164.8,156.8,152.0,150.1,143.6,141.3,139.7,136.8,132.9,128.1,122.6,121.4,115.3,114.1$, 
112.8, 109.0, 89.7, 87.1, 78.5, 76.3, 71.2, 44.4, 43.7, 42.9, 41.4, 34.1, 31.7, 27.4, 24.6, 22.4, 21.9, 20.3, 17.6. FT-IR (neat): 3491, 3372, 2960, 2925, 2872, 1699, 1595, 1461, 1385, 1330, 1176, 1048, 953, 834, 796, $756 \mathrm{~cm}^{-1}$. HRMS-ESI $(\mathrm{m} / \mathrm{z})$ : $[\mathrm{MH}]^{+}$calcd. for $\mathrm{C}_{33} \mathrm{H}_{43} \mathrm{BrNO}_{6} 628.22738$; found 628.22683.

${ }^{1} \mathrm{H}$ NMR (500 MHz, DMSO-d 6 ): $\delta=10.07,(\mathrm{~s}, 1 \mathrm{H}$ ), 7.13 (app. t, $1 \mathrm{H}$ ), 6.87-6.77 (m, $2 \mathrm{H}$ ), 6.466.01 (br. s, $2 \mathrm{H}$ ), 6.36 (d, $1 \mathrm{H}, J=16.4 \mathrm{~Hz}$ ), 6.13 (dt, $1 \mathrm{H}, J=3.2,12.1 \mathrm{~Hz}), 6.06$ (dd, $1 \mathrm{H}, J=7.8$, $15.8 \mathrm{~Hz}$ ), 5.98-5.91 (m, $2 \mathrm{H}), 5.52$ (d, $1 \mathrm{H}, J=4.4 \mathrm{~Hz}), 5.46$ (dd, $1 \mathrm{H}, J=2.0,16.4 \mathrm{~Hz}), 5.22$ (dd, $1 \mathrm{H}, J=9.3,15.0 \mathrm{~Hz}$ ), 5.13-5.01 (m, $2 \mathrm{H}$ ), 4.89 (d, $1 \mathrm{H}, J=4.4 \mathrm{~Hz}$ ), 4.47 (app. t, $1 \mathrm{H}$ ), 3.47-3.37 (m, $1 \mathrm{H}), 2.31-2.18(\mathrm{~m}, 1 \mathrm{H}), 2.05-1.95(\mathrm{~m}, 1 \mathrm{H}), 1.90-1.82(\mathrm{~m}, 1 \mathrm{H}), 1.80-1.68(\mathrm{~m}, 1 \mathrm{H}), 1.45-$ 1.31 (m, 2 H), 1.08-0.99 (m, 2 H), 1.04 (s, 3 H), 0.97 (d, 3 H, $J=7.3$ Hz), 0.95 (s, 3 H), 0.89 (d, 3 $\mathrm{H}, J=6.8 \mathrm{~Hz}$ ), 0.87 (d, $3 \mathrm{H}, J=6.7 \mathrm{~Hz}) .{ }^{13} \mathrm{C}$ NMR (125 MHz, DMSO-d $\left.{ }_{6}\right): \delta=164.2,156.7,153.3$, 151.7, 143.2, 142.5, 139.7, 136.4, 132.0, 126.9, 122.3, 120.1, 114.4, 113.4, 111.7, 106.8, 90.5, $86.4,76.6,75.7,68.3,44.2,43.1,41.8,41.1,33.3,31.3,26.9,24.1,22.4,22.0,19.9,17.5$.

\section{$(2 S, 3 S, 4 E, 6 R, 8 R, 10 R, 12 E)-2$-(Hydroxymethyl)-3,6,10-trimethyl-14-0xooxacyclotetradeca-} 4,12-dien-8-yl carbamate (2)

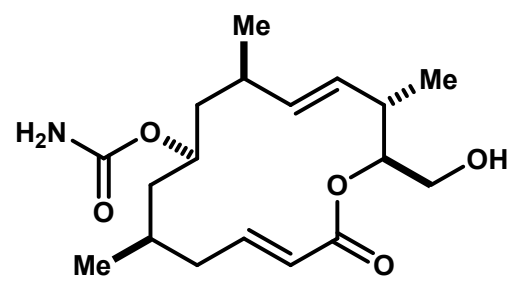

White Solid, $[\alpha]_{\mathrm{D}}=+11.2\left(c=0.1, \mathrm{CHCl}_{3}\right) ;{ }^{1} \mathrm{H}$ NMR $\left(500 \mathrm{MHz}, \mathrm{CDCl}_{3}\right): \delta=7.20(\mathrm{ddd}, 1 \mathrm{H}, J=$ 6.3, 9.2, $15.7 \mathrm{~Hz}), 5.76$ (d, $1 \mathrm{H}, J=15.7 \mathrm{~Hz}), 5.27$ (dd, $1 \mathrm{H}, J=9.8,14.9 \mathrm{~Hz}), 5.02(\mathrm{dd}, 1 \mathrm{H}, J=$ 9.5, 14.9 Hz), 4.87 (app. t, 1 H), 4.59 (br. s., 2 H), 4.54-4.47 (m, 1 H), 3.93 (d, $1 \mathrm{H}, J=12.7 \mathrm{~Hz}$ ), 3.83-3.71 (m, $1 \mathrm{H}), 2.93-2.85$ (m, $1 \mathrm{H}), 2.76-2.66$ (m, $1 \mathrm{H}), 2.45-2.36$ (m, $1 \mathrm{H}), 2.04-1.95$ (m, 1 H), 1.88-1.72 (m, 2 H), 1.57 (dd, $1 \mathrm{H}, J=11.7,14.4, \mathrm{~Hz}$ ), 1.43 (ddd, $1 \mathrm{H}, J=1.4,10.2,14.4 \mathrm{~Hz}$ ), 1.26-1.18 (m, $1 \mathrm{H}), 1.12$ (dd, $1 \mathrm{H}, J=11.7,14.1 \mathrm{~Hz}), 1.02$ (d, $3 \mathrm{H}, J=6.8 \mathrm{~Hz}), 0.97$ (d, $3 \mathrm{H}, J=$ $6.5 \mathrm{~Hz}$ ), 0.91 (d, $3 \mathrm{H}, J=6.8 \mathrm{~Hz}) .{ }^{13} \mathrm{C}$ NMR $\left(125 \mathrm{MHz}, \mathrm{CDCl}_{3}\right): \delta=166.9,157.1,150.0,136.3$, 133.4, 122.2, 81.0, 73.1, 63.2, 44.5, 44.4, 42.5, 38.1, 34.4, 29.7, 24.4, 22.5, 18.1. FT-IR (neat): 
3466, 3362, 2954, 2925, 2871, 1699, 1602, 1382, 1314, 1268, 1205, 1163, 1040, 973, $865 \mathrm{~cm}^{-1}$. HRMS-ESI $(m / z):[M H]^{+}$calcd. for $\mathrm{C}_{18} \mathrm{H}_{30} \mathrm{NO}_{5} 340.21240$; found 340.21185

(3E,7E,10R)-10-(2-Bromo-3-hydroxyphenyl)-10-hydroxy-9,9-dimethyldeca-3,7-dien-5-yn-2yl (E)-but-2-enoate (3)

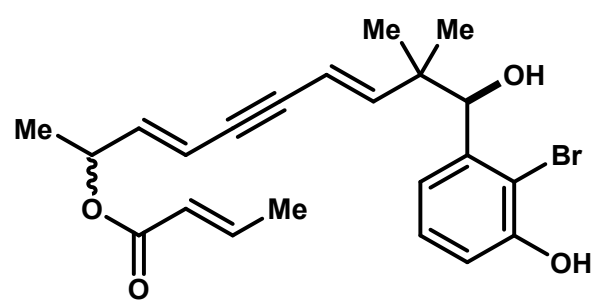

Colorless Oil, ${ }^{1} \mathrm{H}$ NMR (500 MHz, $\mathrm{CDCl}_{3}$ ): $\delta=7.24$ (app. t, $1 \mathrm{H}$ ), 7.06-6.93 (m, $\left.3 \mathrm{H}\right), 6.33$ (d, 1 H, $J=16.3 \mathrm{~Hz}$ ), 6.09 (dd, $1 \mathrm{H}, J=15.9,6.2 \mathrm{~Hz}), 5.90-5.78$ (m, $2 \mathrm{H}), 5.67$ (s, 1H), 5.55 (dd, $1 \mathrm{H}$, $J=16.3,2.1 \mathrm{~Hz}), 5.50-5.42(\mathrm{~m}, 1 \mathrm{H}), 4.99(\mathrm{~d}, 1 \mathrm{H}, J=3.1 \mathrm{~Hz}), 1.93$ (d, $1 \mathrm{H}, J=3.5 \mathrm{~Hz}), 1.88$ (dd, $3 \mathrm{H}, J=6.9,1.6 \mathrm{~Hz}), 1.35(\mathrm{~d}, 3 \mathrm{H}, J=6.6 \mathrm{~Hz}), 1.14(\mathrm{~s}, 3 \mathrm{H}), 1.05(\mathrm{~s}, 3 \mathrm{H}) .{ }^{13} \mathrm{C}$ NMR $(125 \mathrm{MHz}$, $\left.\mathrm{CDCl}_{3}\right): \delta=165.7,151.9,149.9,145.1,141.7,141.2,128.2,122.9,121.5,115.3,112.9,111.4$, 109.1, 89.7, 87.0, 78.6, 69.9, 43.7, 24.5, 22.0, 20.2, 18.1. FT-IR (neat): 3419, 2972, 2932, 2872 , 1698, 1653, 1573, 1440, 1290, 1182, 1101, 1040, 953, 837, 769, $690 \mathrm{~cm}^{-1}$. HRMS-ESI (m/z): [M$\mathrm{H}]^{-}$calcd. for $\mathrm{C}_{22} \mathrm{H}_{24} \mathrm{BrO}_{4} ; 431.08580$; found 431.08634 .

$(2 R, 3 S, 4 E, 6 R, 8 R, 10 R, 12 Z)-2-((E)-B u t-1-e n-3-y n-1-y l)-3,6,10-t r i m e t h y l-14-$ oxooxacyclotetradeca-4,12-dien-8-yl carbamate (S1)

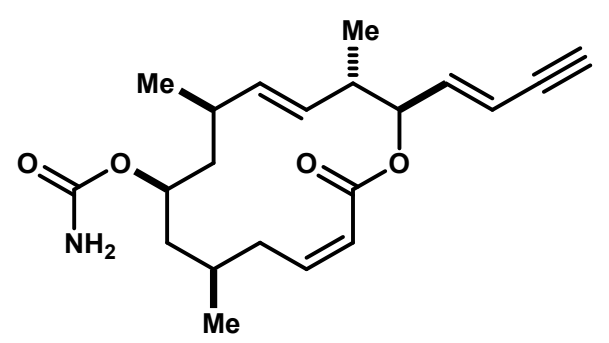

White solid, ${ }^{1} \mathrm{H}$ NMR (500 MHz, $\mathrm{CDCl}_{3}$ ): $\delta=6.18-6.08$ (m, $2 \mathrm{H}$ ), 5.88 (dd, $1 \mathrm{H}, J=11.6,2.3 \mathrm{~Hz}$ ), $5.73(\mathrm{dd}, 1 \mathrm{H}, J=15.9,1.4 \mathrm{~Hz}$ ), 5.30 (dd, $1 \mathrm{H}, J=15.0,9.5 \mathrm{~Hz}), 5.25$ (dd, $1 \mathrm{H}, J=10.0,8.5 \mathrm{~Hz}$ ), 
$5.04(\mathrm{dd}, 1 \mathrm{H}, J=15.0,9.3 \mathrm{~Hz}), 4.64-4.52$ (m, $3 \mathrm{H}), 3.71-3.61(\mathrm{~m}, 1 \mathrm{H}), 2.90$ (d, $1 \mathrm{H}, J=2.3 \mathrm{~Hz})$, 2.25-2.18 (m, 1 H), 2.14-2.04 (m, 1 H), 2.00-1.92 (m, 1 H), 1.92-1.82 (m, 1 H), 1.53-1.43 (m, 2 H), 1.11-1.02 (m, $2 \mathrm{H}), 1.00$ (d, $3 \mathrm{H}, J=7.0 \mathrm{~Hz}), 0.95$ (d, $3 \mathrm{H}, J=6.9 \mathrm{~Hz}), 0.91(\mathrm{~d}, 3 \mathrm{H}, J=6.8$ $\mathrm{Hz}) .{ }^{13} \mathrm{C}$ NMR $\left(125 \mathrm{MHz}, \mathrm{CDCl}_{3}\right): \delta=164.7,156.8,143.8,142.2,136.9,132.7,122.4,112.6$, 81.5, 78.6, 75.8, 71.1, 44.4, 42.8, 41.4, 34.1, 31.7, 27.4, 22.4, 20.3, 17.6. FT-IR (neat): 3492, 3371, $3301,2957,2926,2872,1713,1640,1599,1455,1380,1327,1175,1045,972,835,796,736 \mathrm{~cm}^{-1}$. HRMS-ESI (m/z): [M] $]^{+}$calcd. for $\mathrm{C}_{21} \mathrm{H}_{29} \mathrm{NO}_{4}$ 359.20966; found 359.20911. 